\title{
THE STABILITY INDEX OF A CACTUS
}

\author{
DOUGLAS D. GRANT and D. A. HOLTON \\ (Received 14 May 1976; revised 13 September 1976) \\ Communicated by W. D. Wallis
}

\begin{abstract}
We show that if $\boldsymbol{G}$ is a connected graph of order $\boldsymbol{n}$ such that no line lies in more than one cycle (in other words, $G$ is a cactus of order $n$ ), then the stability index of $G$ is one of the integers $0,1, n-7, n-6, n-5, n-4$ or $n$.

By finding an index-0 graph to which a given cactus optimally reduces. we provide a structural characterization of cacti with given stability index.
\end{abstract}

\section{Introduction}

A cactus is a connected finite graph in which no line lies in more than one cycle. The index- 0 and stable cacti were characterized in McAvaney (1975). In this paper we augment McAvaney's work by proving that the stability index of a cactus of order $n$ is $0,1, n-7, n-6, n-5, n-4$ or $n$, and by giving a characterization of cacti with given stability index.

Throughout this paper, the word graph will mean finite undirected graph with no loops or multiple lines. We shall adopt the basic graph-theoretical terminology of Harary (1969). Notions relating to the stability index of a graph are described in Grant (1974). Since this paper is a sequel to McAvaney (1975), we shall use several ideas introduced in that paper. In particular, we note that $\underline{P}_{n}$ denotes a path $P_{n}$ rooted at an endpoint. For the purposes of the present paper we require also the following definitions, in which $G$ denotes an arbitrary graph.

The graph $H$ is a semi-stable extension of $G$, if $H$ has a partial stabilizing sequence $S$ such that $H_{S}=G$. (Note that this differs slightly from the definition of "semistable extension" given in Grant, 1974.) We say that $H$ is reducible to $G$, or that $H$ reduces to $G$ if $H$ is a semi-stable extension of $G$, and that $H$ is optimally reducible to $G$, or $H$ optimally reduces to $G$, if $H$ is reducible to $G$ and s.i. $(H)=|V(H)|-|V(G)|$. If $H$ is a semi-stable extension of $G$ such that $|V(H)|-|V(G)|=k$, we say that $H$ is a rank- $k$ extension of $G$. If the points $u_{1}, u_{2}, \ldots, u_{m}$ form, in order, a partial stabilizing sequence for $G$, we shall denote this sequence by $: u_{1}, u_{2}, \ldots, u_{m}:$.

We shall adopt the convention that whenever we need to label specifically the points of the path $P_{n}$, we shall use the labels $v_{1}, v_{2}, \ldots, v_{n}$, where $v_{i}$ is adjacent to $v_{i+1}$ for $i=1,2, \ldots, n-1$. 
A cactus $C$ is said to contain an essential copy of $P_{n}(n>1)$ if it can be formed from $P_{n}$ by one or both of the following constructions:

(i) for some integer $i, 1 \leqslant i \leqslant \frac{1}{2} n$, join a new point $b$ to points $v_{i}$ and $v_{n-i+1}$ of $P_{n}$, and root an arbitrary (possibly trivial) cactus at $b$;

(ii) if $n$ is odd, root an arbitrary cactus at $v_{\frac{1}{2}(n+1)}$.

For example, the cacti which contain an essential coppy of $P_{5}$ are of the forms shown in Fig. 1.

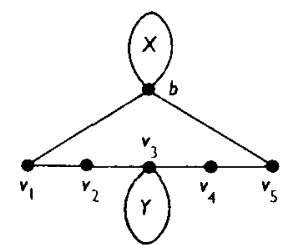

(a)

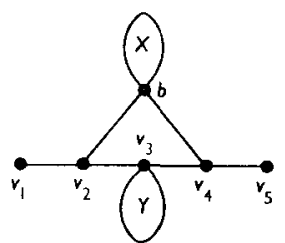

(b)

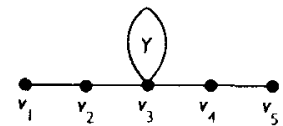

(c)

Cacti with an essential copy of $\boldsymbol{P}_{5}$.

$X$ denotes a cactus rooted at $b ; Y$ denotes a cactus rooted at $v_{3}$.

FIG. 1.

The copy of $\boldsymbol{P}_{n}$ from which $C$ is formed by the above construction is said to be an essential copy of $P_{n}$. (Note that in the terminology of McAvaney (1975) a transfig in a cactus corresponds to an essential copy of either $P_{2}$ or $P_{3}$.)

An essential copy of $P_{n}$ in a rooted cactus $C$ is an essential copy of $P_{n}$ in the underlying unrooted cactus, which does not contain the root of $C$.

For $n=2,3,4,5$ and 6 , let $d_{n}(C)$ denote the maximum number of (point-) disjoint essential copies of $P_{n}$ in the cactus $C$.

The following results will be of use to us in subsequent sections. Here and henceforth the symbol \# will be used to denote the end of, or absence of, a proof.

LEMMA 1 (Holton and Grant, 1975). The graph $G$ is semi-stable at point $v$ if and only if the set of points of $G$ adjacent to $v$ is fixed by the automorphism group of $G_{v} . \quad \#$

LEMma 2 (McAvaney, 1975). A rooted cactus $C$ is semi-stable at a point which is neither a cutpoint nor the root. \#

In proving Lemma 2, McAvaney essentially provided the following algorithm to find a point at which a rooted cactus $C$ is semi-stable. The terminology we use in our description of the algorithm is that introduced in McAvaney (1975).

\section{MCAvaney's Algorithm}

Step 1. Let $b_{1}$ denote the root of $C$, and set $i=1$.

Step 2. Let $B_{i}$ be a smallest branch at $b_{i}$ which, if $i>1$, does not contain $b_{i-1}$, and let $D_{i}$ denote the block in $B_{i}$ that contains $b_{i}$. If either $i=1$ and $D_{i}$ has no cutpoints, or $i>1$ and $b_{i}$ is the only cutpoint in $D_{i}$, let $w$ be any point in $B_{i}$ adjacent to $b_{i}$, and go to Step 7 . 
Step 3. Let $b_{i+1}^{*}$ be a cutpoint in $D_{i}$ closest to $b_{i}$ such that the branch $B_{i+1}^{*}$ at $D_{i}$ containing $b_{i+1}^{*}$ is as small as possible. If $B_{i+1}^{*}$ is not $\underline{P}_{2}$, relabel $b_{i+1}^{*}$ as $b_{i+1}$, and go to Step 6.

Step 4. Let $a$ be the endpoint in $B_{i+1}^{*}$. If $C$ is semi-stable at $a$, set $w=a$ and go to Step 7.

Step 5. If $b_{i}$ and $b_{i+1}^{*}$ are the only cutpoints in $D_{i}$, then $D_{i}$ is a cycle; in this case, $C$ is semi-stable at some point $w$ in $D_{i}$ adjacent to $b_{i}$ or $b_{i+1}^{*}$. Go to Step 7. Otherwise, let $b_{i+1}$ be a cutpoint in $D_{i}$, next closest after $b_{i+1}^{*}$ to $b_{i}$ such that the branch at $D_{i}$ containing $b_{i+1}$ is as small as possible.

Step 6. Increase $i$ by 1 , and then go to Step 2 .

Step 7. $C$ is a semi-stable at $w$. Terminate algorithm. \#

The following theorem is an amalgamation of results of Robertson and Zimmer (1972), Heffernan (1972), McAvaney, Grant and Holton (1974) and McAvaney (1975).

THEOREM 1. The index-0 cacti are $P_{n}$ for all $n \geqslant 4$ and the graphs $E_{7}, U_{1}, U_{2}, U_{3}$, $U_{4}$ and $U_{5}$ shown in Fig. 2. A semi-stable cactus is semi-stable at a point which is either a penultimate point or a non-cutpoint. \#
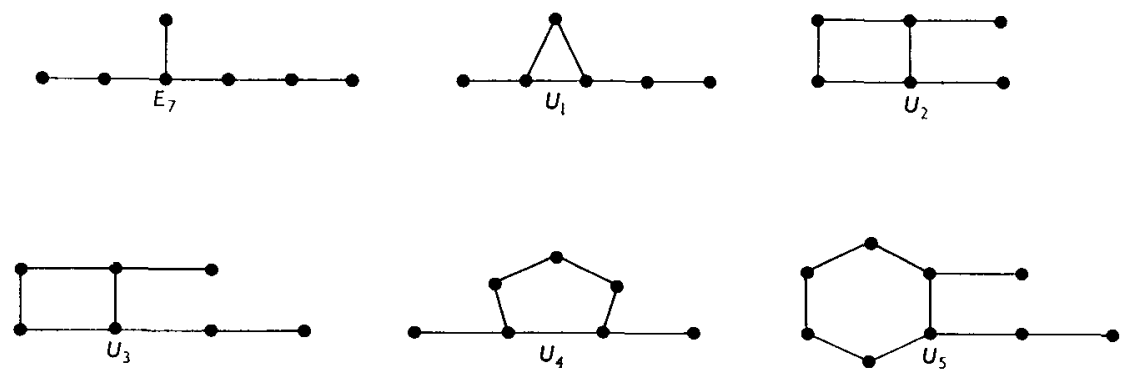

FIG. 2. Some index-0 cacti.

Lemma 3 (Grant, 1974). Let $T$ be a tree of order $n$. Then s.i. $(T)=0,1, n-7$, $n-5$ or $n$, and

(i) s.i. $(T)=n$ if and only if either $T \cong K_{1}$ or $d_{2}(T)+d_{3}(T)>0$,

(ii) s.i. $(T)=n-5$ if and only if $d_{2}(T)+d_{3}(T)=0$ and $d_{5}(T)>0$,

(iii) s.i. $(T)=n-7$ if and only if $d_{2}(T)+d_{3}(T)+d_{5}(T)=0$ and $T \not P_{n}$ for any $n>3$ other than $n=7$.

If s.i. $(T)=n-5$, then $T$ is optimally reducible to $P_{5}$, and if $\mathrm{s.i.}(T)=n-7$, then $T$ is optimally reducible to $E_{7}$ unless $T \cong P_{7}$. \#

As mentioned previously, it is our chief goal in this paper to extend the result of Lemma 3 to cover all cacti. In McAvaney (1975), the first step was completed when the following result was proved. 
TheOREM 2 (McAvaney, 1975). Let $C$ be a cactus of order $n$. Then s.i. $(C)=n$ if and only if either $C \cong K_{1}$ or $d_{2}(C)+d_{3}(C)>0$. (Equivalently, $C$ is stable if and only if either $C \cong K_{1}$ or the automorphism group of $C$ contains a transposition.) \#

\section{The stability index of cacti}

In this section we prove

THEOREM 3. If $C$ is a cactus of order $n$, then s.i. $(C)=0,1, n-7, n-6, n-5$, $n-4$, or $n$.

It is convenient to break the proof of Theorem 3 into a number of steps which we describe in the lemmas to follow. Of these lemmas, we detail the proof of only one. The others can be proved by using similar techniques.

First of all, Theorem 1 implies

LEMMA 4. If $C$ is an unstable cactus, then $C$ is reducible to an index-0 cactus. \# \#

LEMMA 5. If $n \geqslant 9$, the only cacti which are optimally reducible to $P_{n}$ are $P_{n}$ and $C_{n+1}$. The only cacti which are optimally reducible to $P_{8}$ are $P_{8}, C_{9}$ and the graph $U_{6}$ shown in Fig. 3. All other unstable cacti which are reducible to $P_{n}$, for $n \geqslant 8$, are reducible to either $E_{7}$ or $U_{1}$.
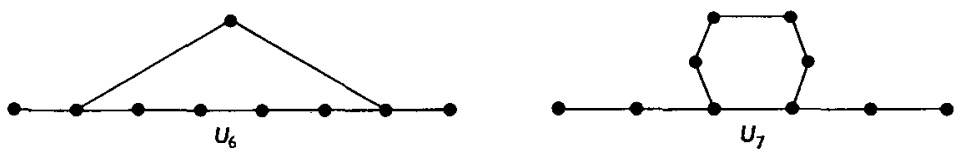

Fig. 3.

Proof. Suppose that $n \geqslant 8$ and that $C$ is a cactus which is reducible to $P_{n}$. If $C \cong P_{n}$ or $C \cong C_{n+1}$, then there is nothing to prove. Let us therefore suppose that $C \nsupseteq P_{n}, C \nsupseteq C_{n+1}$. Let $S=: u_{1}, u_{2}, \ldots, u_{m}$ : be a partial stabilizing sequence for $C$ such that $C_{S} \cong P_{n}$, and let $K=C_{u_{1}, u_{2}, \ldots, u_{m-1}}$. Since $K$ is a rank-1 extension of $P_{n}$, it follows from Lemma 1 that either (i) there is $i$ with $1 \leqslant i \leqslant\left\{\frac{1}{2} n\right\}$ such that $N_{K}\left[u_{m}\right]=\left\{v_{i}, v_{n-i+1}\right\}$ or (ii) $N_{K}\left[u_{m}\right]=\varnothing$.

Case (i). $N_{K}\left[u_{m}\right]=\left\{v_{i}, v_{n-i+1}\right\}$. If $i=1$, then $K \cong C_{n+1}$. By Lemma 1, since $C_{n+1}$ is point-transitive it follows that in any connected semi-stable extension of $K$ there is a point which is adjacent to all the points of $K$. We deduce that the only cactus which is a semi-stable extension of $K$ is $K$ itself, so that $C=K \cong C_{n+1}$, in contradiction to our hypothesis.

If $n$ is odd and $i=\left[\frac{1}{2} n\right]$, then $K$ contains an essential copy of $P_{3}$, and so is stable by Theorem 2. In this case $C$, being a semi-stable extension of $K$, is stable.

If $n$ is odd and $i=\left\{\frac{1}{2} n\right\}$, then $K$ is a tree, which by Lemma 3 is optimally reducible to $E_{7}$. It follows that $C$ is reducible to $E_{7}$. 
If $n=8$ and $i=2$, then $K \cong U_{6}$. By inspection, $U_{6}$ is optimally reducible to $P_{8}$. It follows that if $m=1$, then $C \cong U_{6}$ and is optimally reducible to $P_{8}$. Now suppose that $m>1$. Let $L=G_{u_{1}, u_{2}, \ldots, u_{m-8}}$. Since $L$ is a rank-1 extension of $U_{6}$, it follows from Lemma 1 that either $N_{L}\left[u_{m-1}\right]=\left\{u_{m}\right\}$ or $N_{L}\left[u_{m-1}\right]=\emptyset$. In the former case $L$ is reducible to $E_{7}$, so that $C$ is reducible to $E_{7}$. If $N_{L}\left[u_{m-1}\right]=\varnothing$, then $m>2$. Let $M=C_{u_{1}, u_{2} \ldots, u_{m-3}}$. Since $M$ is a rank-1 extension of $L$, it follows from Lemma 1 that either $N_{M}\left[u_{m-2}\right]=\varnothing$ or $N_{M}\left[u_{m-2}\right]=\left\{u_{m-1}\right\}$ or $N_{M}\left[u_{m-2}\right]=\left\{u_{m-1}, u_{m}\right\}$ or $N_{M}\left[u_{m-2}\right]=\left\{u_{m}\right\}$. In the first two cases $M$ has a transposition automorphism, and so $C$, being a semi-stable extension of $M$, also has a transposition automorphism. By Theorem 2, $C$ is stable. In the remaining two cases $M$ is, by inspection, reducible to $E_{7}$, and so $C$ is reducible to $E_{7}$.

Finally, if $i>1, n \geqslant 8$, and $i \neq 2$ if $n=8, i \neq\left[\frac{1}{2} n\right]$ or $\left\{\frac{1}{2} n\right\}$ if $n$ is odd, then $K$ is a unicyclic graph which can readily be shown to reduce to either $U_{1}$ or $E_{7}$. In such cases, $C$ is therefore reducible to either $U_{1}$ or $E_{7}$.

Case (ii). $N_{K}\left[u_{m}\right]=\varnothing$. In this case $m \geqslant 2$. Let $L=C_{u_{1}, u_{2}, \ldots, u_{m-2}}$. Since $L$ is a rank-1 extension of $K$, it follows by Lemma 1 that either there is $i$ with $1 \leqslant i \leqslant\left\{\frac{1}{2} n\right\}$ such that $N_{L}\left[u_{m-1}\right]=\varnothing$ or $N_{L}\left[u_{m-1}\right]=\left\{u_{m}\right\}$ or $N_{L}\left[u_{m-1}\right]=\left\{v_{i}, v_{n-i+1}, u_{m}\right\}$ or $N_{L}\left[u_{m-1}\right]=$ $\left\{v_{i}, v_{n-i+1}\right\}$. In the first two cases $L$ has a transposition automorphism, so that $C$ has a transposition automorphism. By Theorem 2, $C$ is stable in such cases. If $N_{L}\left[u_{m-1}\right]=\left\{v_{i}, v_{n-i+1}, u_{m}\right\}, L$ can readily be shown to reduce to either $U_{1}$ or $E_{\eta}$. If $N_{L}\left[u_{m-1}\right]=\left\{v_{i}, v_{n-i+1}\right\}$, let $M=C_{u_{1}, u_{2}, \ldots, u_{m-2}}$. Since $M$ is a rank-1 extension of $L$, it follows from Lemma 1 that $N_{M}\left[u_{m-2}\right]=\varnothing,\left\{u_{m}\right\},\left\{u_{m-1}\right\}$, or $\left\{u_{m-1}, u_{m}\right\}$. In the first two cases $M$, and hence $C$, has a transposition automorphism and therefore $C$ is stable. In the remaining two cases $M$ is readily shown to be reducible to $U_{1}$ or E. \#

LEMMA 6. The only cacti which are optimally reducible to $U_{5}$ are $U_{5}$ and the graph $U_{7}$ shown in Fig. 3. All other unstable cacti which are reducible to $U_{5}$ are also reducible to $E_{7}$. \#

The next result, of a similar nature to the preceding two, will be used in the following section.

LEMMA 7. All cacti other than $P_{7}$ and $C_{8}$ which are optimally reducible to $P_{7}$ are also optimally reducible to $E_{7}$. All cacti other than $U_{4}$ which are optimally reducible to $U_{4}$ are also optimally reducible to $E_{7}$. \#

We now return to Theorem 3 .

Proof of Theorem 3. That there are cacti of order $n$ with stability index equal to 0,1 and $n$ is clear from Theorems 1 and 2 and Lemma 5. Suppose that $C$ is a cactus of order $n$ such that s.i. $(C) \notin\{0,1, n\}$. It follows that $C \nsupseteq C_{n}, C \nsubseteq U_{6}, C \nsubseteq U_{7}$. 
By Lemma 4, $C$ is reducible to an index-0 cactus $C^{*}$. By Theorem $1, C^{*}$ is $P_{k}$ for some $k \geqslant 4, E_{7}, U_{1}, U_{2}, U_{3}, U_{4}$ or $U_{5}$. By Lemmas 5 and 6 and the fact that s.i. (C) $\notin\{1, n\}$, it follows that if $C^{*}$ is $P_{k}$ for some $k \geqslant 8$, or $U_{5}$, then $C$ is reducible to $E_{7}$ or to $U_{1}$. We deduce that $C$ is reducible to $P_{4}, P_{5}, P_{6}, P_{7}, E_{7}, U_{1}, U_{2}, U_{3}$ or $U_{4}$, so that s.i. $(C) \geqslant n-7$. Since $C$ is not stable, and because all graphs of order less than 4 are stable, it follows that s.i. $(C) \leqslant n-4$. We deduce that s.i. $(C)=n-7$, $n-6, n-5$ or $n-4$. \#

Remark. The results of the next section show that none of the possibilities $0,1, n-7, n-6, n-5, n-4$ or $n$ can be disposed of in the statement of Theorem 3 .

\section{Characterization of cacti with given stability index}

For the purpose of this section it is convenient to introduce some new terminology and notation. For $k=4,5,6$, or 7 let $A_{k}$ denote the set of all cacti optimally reducible to an index- 0 graph on $k$ points. Let $A_{0}$ denote the set of all stable cacti, $A$ the set of all index-0 cacti, and $U=\left\{C_{n}: n \geqslant 9\right\} \cup\left\{U_{6}, U_{7}\right\}$.

It follows from Lemmas 4,5 and 6 that $U$ is the set of all index-1 cacti on more than 8 points. Hence Theorem 3 implies that $A_{0} \cup A_{4} \cup A_{5} \cup A_{6} \cup A_{7} \cup A \cup U$ contains all cacti. In this section it is our goal to describe how the structure of a particular cactus determines to which of the sets $A_{0}, A_{4}, A_{5}, A_{6}, A_{7}, A, U$ the cactus belongs. By doing so, we will characterize cacti with a given stability index. We have already characterized the elements of $A$ (Theorem 1) and $A_{0}$ (Theorem 2). Since the elements of $U$ have been explicitly listed, our task is to characterize the elements of $A_{4}, A_{5}$, $A_{6}$ and $A_{7}$. We note that $A \cap A_{4}=\left\{P_{4}\right\}, A \cap A_{5}=\left\{P_{5}\right\}, A \cap A_{6}=\left\{U_{1}, U_{2}, P_{6}\right\}$, $A \cap A_{7}=\left\{U_{3}, U_{4}, P_{7}, E_{7}\right\}, A_{i} \cap U=\varnothing$, for $i=4,5,6$ and $7, A \cap U=\varnothing$ and that $A_{i} \cap A_{j}=\varnothing$ for $4 \leqslant i<j \leqslant 7$.

First of all we shall characterize the elements of $A_{4}$.

Lemma 8. If $C \in A_{4}$, then $d_{4}(C)>0$.

Proof. If $C \in A_{4}$, then $C$ optimally reduces to an index-0 graph of order 4. Since the only index-0 graph of order 4 is $P_{4}, C$ reduces to $P_{4}$, and so $C$ is a semi-stable extension of $P_{4}$. This implies that $C$ contains an essential copy of $P_{4}$, that is, $d_{4}(C)>0$. \#

Lemma 9. If $C$ is a cactus such that $d_{4}(C)=1$, then $C$ is reducible to $P_{4}$.

Proof. If $C \cong P_{4}$ there is nothing to prove. Let us suppose that $C \nsucceq P_{4}$. It follows that $C$ is one of the forms (a), (b) shown in Fig. 4. The two cases must be dealt with separately. As similar techniques are used in the different cases, we shall only prove here that a cactus of form (a) is reducible to $P_{4}$, this being the more involved of the two cases. 


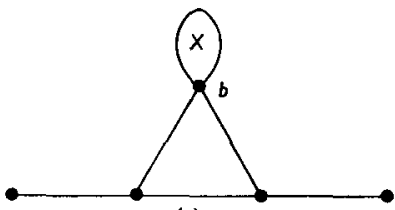

(o)

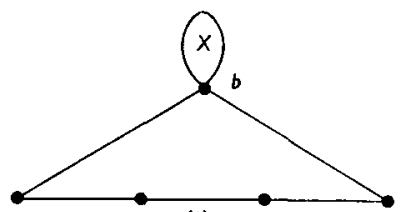

(b)

FIG. 4. Cacti with an essential copy of $P_{4} . X$ denotes a cactus rooted at $b$.

Thus suppose that $C$ is of form (a) and let $|V(C)|=n$. We shall prove that $C$ is reducible to $P_{4}$ by induction on $n$. Note that $n \geqslant 5$. If $n=5$, then $X_{b}$ is null and $C$ is semi-stable at $b$. Since in this case $C_{b} \cong P_{4}$, it follows that $C$ is reducible to $P_{4}$. If $n=6$, then $C$ is semi-stable at the unique point $u$ of $X_{b}$ and $C_{u}$ is semi-stable at $b$. Since $C_{u, b} \cong P_{4}$, again $C$ is reducible to $P_{4}$. If $n=7$, then $C$ is one of the cacti $U_{8}, U_{9}, U_{10}$ shown in Fig. 5. It is a trivial exercise to show that each of $U_{8}, U_{9}, U_{10}$ is reducible to $P_{4}$, so that if $n=7, C$ is reducible to $P_{4}$.
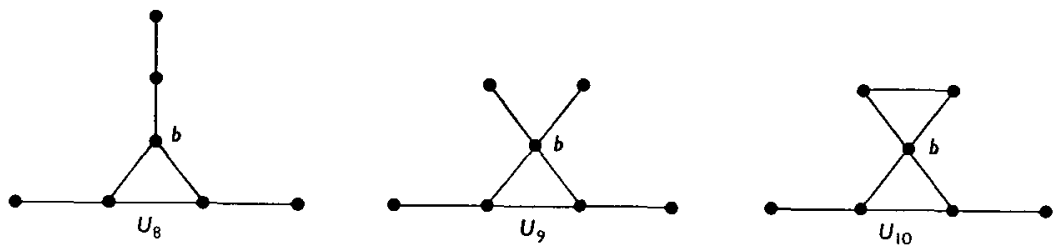

FIO. 5.

Now suppose that $n>7$, and that the result holds for all cacti under consideration which have order $<n$. Let us consider $X$ to be a rooted cactus with root $b$. By Lemma 2, $X$ is semi-stable at a point $w \neq b$ which is not a cutpoint. Since $d_{4}(C)=1$, it follows that $d_{4}(X)=0$, and so $d_{4}\left(X_{v}\right)=0$. We deduce that $d_{4}\left(C_{w}\right)=1$, the essential copy of $P_{4}$ in $C_{w}$ having the same points as the essential copy of $P_{4}$ in $C$. Since $n>7, X_{w} \supsetneqq P_{2}$, and so $C$ is semi-stable at $w$. Now $C_{w}$ is a cactus of order $n-1$ with $d_{4}\left(C_{w}\right)=1$. By the inductive hypothesis, $C_{w}$ is reducible to $P_{4}$. It follows that $C$ is reducible to $P_{4}$.

The lemma now follows by induction. \#

Lemma 10. If $C$ is a cactus such that $d_{4}(C)>0$, then $C$ is reducible to $P_{4}$.

Proof. We shall prove the lemma by induction on $|V(C)|$. The lemma is true if $|V(C)|=4$. (Here $C \cong P_{4}$, and is the smallest cactus which has an essential copy of $P_{4}$.) Suppose, then, that $|V(C)|=n>4$ and that the lemma holds for all cacti under consideration which have order $<n$. If $d_{4}(C)=1$, then $C$ is reducible to $P_{4}$ by Lemma 9 . We shall therefore suppose that $d_{4}(C)>1$. By Theorem $1, C$ is semi-stable at some point $w$ which is either a non-cutpoint or a penultimate point. 
In the former case, $C_{w}$ is a cactus of order $n-1$, with $d_{4}\left(C_{w}\right) \geqslant 1$. By the inductive hypothesis, $C_{w}$ is reducible to $P_{4}$, and so $C$ is reducible to $P_{4}$. In the latter case, $C_{w}$ is semi-stable at the endpoint $x$ of $C$ which is adjacent to $w$, and $C_{v x}$ is a cactus of order $n-2$ with $d_{4}\left(C_{w, x}\right) \geqslant 1$. By the inductive hypothesis, $C_{w, x}$ is reducible to $P_{4}$, and so $C$ is reducible to $P_{4}$. The lemma therefore holds by induction. \#

From Theorem 2, Lemma 8 and Lemma 10, we deduce

THEOREM 4. The cacti in $A_{4}$ are precisely those cacti $C$ such that $\sum_{i=2}^{3} d_{i}(C)=0$ and $d_{4}(C)>0$. \#

We now consider the cacti in $A_{5}$. Basically we follow the same steps as those traced out above in characterizing the cacti in $A_{4}$. Thus our first step is to prove a result corresponding to Lemma 8 . It is indeed straightforward to show that if $C \in A_{5}$, then $C$ contains an essential copy of $P_{5}$. However, consider the cactus $H$ shown in Fig. 6. This cactus contains an essential copy of $P_{5}$, but is only semi-stable at points $v_{1}, v_{2}, v_{4}$ and $v_{5}$, and consequently is not reducible to $P_{5}$. To take into account graphs like $H$ which resist a straightforward paralleling of the steps used to prove Theorem 4 , we proceed as follows.
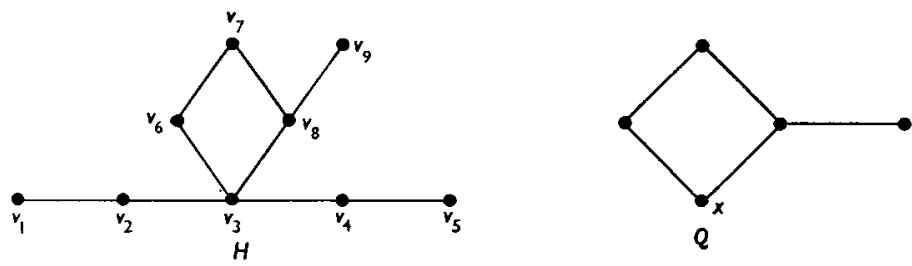

FIG. 6.

Let us say that an essential copy of $P_{5}$ of the form of Fig. 1(c) in a (rooted or unrooted) cactus is strict if no branch of $Y$ at $v_{3}$ is isomorphic to the rooted cactus $Q$, with root $x$, shown in Fig. 6 . Let $d_{5}(C)$ denote the maximum number of point disjoint strict essential copies of $P_{5}$ in the cactus $C$.

LEMMA 11. If $C$ is a (rooted or unrooted) cactus such that $d_{5}(C)=0$, and $C$ is reducible to the cactus $C^{*}$, then $\bar{d}_{5}\left(C^{*}\right)=0$.

Proof. If $C$ has no essential copies of $P_{5}$, it is clear that $C^{*}$ has no essential copies of $P_{5}$. In this case the lemma holds. Let us suppose, then, that $C$ has essential copies of $P_{5}$ (which are not strict), and that $C^{*}$ has the strict essential copy of $P_{5}$ whose successively adjacent points are $v_{1}, v_{2}, v_{3}, v_{4}, v_{5}$. Then $\left\langle\left\{v_{1}, v_{2}, v_{3}, v_{4}, v_{5}\right\}\right\rangle$ is an essential copy of $P_{5}$ in $C$ which, by hypothesis, is not strict. It follows that $C$ has a branch at $v_{3}$ isomorphic to $Q$. Suppose that the points of this branch are $v_{3}, v_{6}$, $v_{7}, v_{8}$ and $v_{9}$, as shown on $H$ in Fig. 6 . Let $S$ be a partial stabilizing sequence for 
$C$ such that $C_{S}=C^{*}$. In reducing $C$ to $C^{*}$ via $S$, at least one of the points $v_{6}, v_{7}$ $v_{8}$ and $v_{9}$ is removed. However, removing any one of $v_{6}, v_{7}, v_{8}, v_{9}$ creates new automorphisms, contrary to the fact that $S$ is a partial stabilizing sequence. This contradiction invalidates the hypothesis that $d_{5}\left(C^{*}\right)>0$. \#

REMARK. Lemma 11 formalizes and generalizes the reason why the graph $H$ of Fig. 6 fails to reduce to $P_{5}$.

Lemma 12. If $C \in A_{5}$, then $d_{5}(C)>0$.

Proof. Since $C \in A_{5}, C$ reduces to an index-0 graph of order 5 , which must be $P_{5}$. Since $d_{5}\left(P_{5}\right)>0$, Lemma 11 shows that $d_{5}(C)>0$. \#

LemMA 13. If $C$ is a cactus such that $\bar{d}_{5}(C)=1$, then $C$ is reducible to $P_{5}$.

Proof. $C$ is of one of the forms of Fig. 1, where, if $C$ is of form (c), no branch at $v_{3}$ is isomorphic to $Q$. The three cases must be dealt with separately. However, we include here only the details for $C$ of the forms (a) or (c), the proof for (b) being similar to that of (a).

I. First of all, suppose that $C$ is of form (a). We may suppose without loss of generality that $|V(X)| \leqslant|V(Y)|$. We shall prove that $C$ is reducible to $P_{5}$ by induction on $n=|V(C)|$. Note that $n \geqslant 6$. If $n=6$, then $C \cong C_{6}$, and $C$ is reducible to $P_{5}$. Suppose that $n>6$, and that all cacti under consideration of order $<n$ are reducible to $P_{5}$. If $X_{b}$ is non-null, then by Lemma $2, X$ is semi-stable at a noncutpoint $w \neq b$. Since $|V(X)| \leqslant|V(Y)|, X_{w} \nsubseteq Y$, and it follows that $C$ is semi-stable at $w$. By the inductive hypothesis, $C_{w}$ is reducible to $P_{5}$, and so $C$ is reducible to $P_{5}$. Now suppose that $X_{b}$ is null. Since $n>6, Y_{v_{8}}$ is non-null. By Lemma 2, $Y$ is semistable at a non-cutpoint $x \neq v_{3}$. Since $d_{5}(C)=1$, we have $d_{5}\left(Y_{v_{3}}\right)=0$, and $C$ is semi-stable at $x$ unless either

$$
\text { (i) } V(Y)=\left\{v_{3}, x\right\} \quad \text { or } \quad \text { (ii) } Y_{x}^{*} \cong C_{6} \text {, }
$$

where $Y^{*}$ is the smallest branch of $Y$ at $v_{3}$, considered here as an unrooted graph. If $C$ is semi-stable at $x$, then by the inductive hypothesis $C_{x}$ is reducible to $P_{5}$, and so $C$ is reducible to $P_{5}$. If $C$ is not semi-stable at $x$, and (i) is the case, then : $v_{3}, x$ : is a partial stabilizing sequence for $C$, and $C_{v_{3}, x} \cong P_{5}$. If $C$ is not semi-stable at $x$ and (ii) is the case, then the subgraph of $C$ induced by $\left\{v_{1}, v_{2}, v_{3}, v_{4}, v_{5}\right\} \cup V\left(Y^{*}\right)$ has two disjoint strict essential copies of $P_{5}$, and so $d_{5}(C)>1$, which is a contradiction. We see that in all cases $C$ is reducible to $P_{5}$, and so the lemma holds for cacti of form (a) by induction.

II. Now suppose that $C$ is of form (c). Once more we shall prove that $C$ is reducible to $P_{5}$ by employing induction on $n=|V(C)|$. Note that $n \geqslant 5$. If $n=5$, then $C \cong P_{5}$, and there is nothing to prove. Suppose, then, that $n>5$, and that all 
cacti under consideration of order $<n$ are reducible to $P_{5}$. Since $n>5, Y_{v_{3}}$ is non-null. By Lemma 2, $Y$ is semi-stable at a non-cutpoint $w \neq v_{3}$. Let us suppose $w$ to be the vertex of semi-stability selected by McAvaney's algorithm.

Since $d_{5}(C)=1$, we deduce that $\bar{d}_{5}(Y)=0$ and so $\bar{d}_{5}\left(Y_{w}\right)=0$. It follows that if $C$ is not semi-stable at $w$, then the only automorphisms of $C_{w}$ which do not extend to automorphisms of $C$ must map a branch of $Y_{w}$ at $v_{3}$ which is isomorphic to $\underline{P}_{3}$ onto either $\left\langle\left\{v_{1}, v_{2}, v_{3}\right\}\right\rangle$ or $\left\langle\left\{v_{3}, v_{4}, v_{5}\right\}\right\rangle$. We deduce that $Y_{w}$ has a branch at $v_{3}$ isomorphic to $\underline{P}_{3}$. Let us suppose that the successively adjacent points of this branch are $v_{3}, u_{1}, u_{2}$ (see Fig. 7), and that $\left\langle V(Y) \backslash\left\{u_{1}, u_{2}, w\right\}\right\rangle$ is denoted by $Z$.

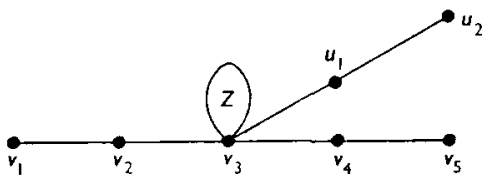

FIG. 7. Part of $C_{w \text {. }}$

In $C, w$ is adjacent to either or both of $u_{1}, u_{2}$, and so there is a path $P$ in $C$ joining $v_{3}$ and $w$ which includes at least one of $u_{1}, u_{2}$. We shall now consider three cases which exhaustively cover all possibilities. In each, let $B$ denote the branch of $C$ at $v_{3}$ which contains $w$, and $D$ the block of $B$ which contains $w$.

$(\alpha)$ There is no path in $C$ joining $v_{3}$ and $w$ which is internally disjoint from $P$. In this case $w \sim v_{3}$. Since $w$ is not a cutpoint of $C$, it follows that $N_{C}[w] \subseteq\left\{u_{1}, u_{2}\right\}$. If $u_{1} \in N_{C}[w]$, then $: u_{1}, u_{2}, w$ : is a partial stabilizing sequence for $C$, and $C_{u_{1}, u_{2}, w}$ is reducible to $P_{5}$ by the inductive hypothesis. If $N_{C}[w]=\left\{u_{2}\right\}$, then $: u_{2}, w:$ is a partial stabilizing sequence for $C$, and $C_{u_{2}, w}$ is reducible to $P_{5}$ by the inductive hypothesis. In either case, $C$ is reducible to $P_{5}$.

( $\beta) ~ w \sim v_{3}$.

Since $C$ is a cactus, there is no path in $C$ joining $v_{3}$ and $w$ which is internally disjoint from both $P$ and the path consisting of the line joining $w$ and $v_{3}$. Since $w$ is not a cutpoint, it follows that $N_{C}[w] \subseteq\left\{v_{3}, u_{1}, u_{2}\right\}$. Arguing as in case $(\alpha)$ we find that we can always reduce $C$ to $P_{5}$.

$(\gamma) w \sim v_{3}$, and there is a path $R$ in $C$ joining $v_{3}$ and $w$ which is internally disjoint from $P$.

Note that in this case we cannot have both $w \sim u_{1}, w \sim u_{2}$. Suppose first of all that $w \sim u_{2}$. Inspection of McAvaney's algorithm shows that $w$ could not have been selected at Step 2 or Step 4. It follows that $w$ was selected at Step 5, so that $D$ is a cycle, the only cutpoints of $C$ which lie in $D$ being $v_{3}$ and the point $x$, say, of $D$ other than $u_{2}$ which is adjacent to $w$. Further, the branch on $D$ at $x$ is isomorphic to $\underline{P}_{2}$. Now $C$ is semi-stable at $u_{1}$ (see Fig. 8(a)), and $C_{u_{1}}$ is reducible to $P_{5}$ by the inductive hypothesis. It follows that $C$ is reducible to $P_{5}$. 


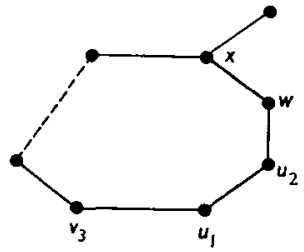

(a)

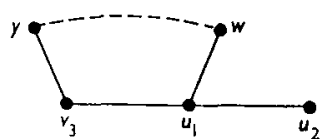

(b)

FIg. 8. (a) Part of $Y$ in case $(\gamma)$, with $w \sim u_{2}$. (b) Part of $Y$ in case $(\gamma)$, with $w \sim u_{1}$.

Now suppose that $w \sim u_{1}$. Here $u_{1}$ is a closest cutpoint in $B$ to $v_{3}$, and the branch at $u_{1}$ which does not contain $v_{3}$ is isomorphic to $\underline{P}_{2}$. Since McAvaney's algorithm selected $w$ rather than $u_{2}$ as the point of semi-stability of $Y$, it follows that Step 5 of the algorithm was executed. Further, $w$ could only be selected at Step 5 if $u_{1}$ was the only cutpoint of $B$ in $D$. We deduce that $D$ is a cycle, and, since no branch of $C$ at $v_{3}$ is isomorphic to $Q,|V(D)| \geqslant 5$. Let $y$ denote the point of $D$ other than $u_{1}$ which is adjacent to $v_{3}$. Then $C$ is semi-stable at $y$ (see Fig. $8(b)$ ), and $C_{y}$ is reducible to $P_{5}$ by the inductive hypothesis. It follows that $C$ is reducible to $P_{5}$.

All cases having been covered, the lemma holds for cacti of form (c) by induction. \#

The proof of the following lemma is similar to that of Lemma 10.

Lemma 14. If $C$ is a cactus such that $d_{5}(C)>0$, then $C$ is reducible to $P_{5}$. \#

From Lemmas 12 and 14 and Theorems 2, 3 and 4 we deduce

THEOREM 5. The cacti in $A_{5}$ are precisely those cacti $C$ such that $\sum_{i=2}^{4} d_{i}(C)=0$ and $d_{5}(C)>0$. \#

We now characterize the cacti in $\boldsymbol{A}_{\mathbf{6}}$.

LEMMA 15. If $C \in A_{6}$, then either $C \cong U_{2}$, or $U_{1} \subseteq C$ or $d_{6}(C)>0$.

Proof. If $C \in A_{6}$, then $C$ reduces to an index-0 graph of order 6. All such graphs are connected, and so $C$ reduces to an index-0 cactus of order 6 . Thus $C$ reduces to $U_{2}, U_{1}$ or $P_{6}$. Since $U_{2}$ is the only cactus which is a semi-stable extension of $U_{2}$, it follows that if $C \nsubseteq U_{2}$, then $C$ reduces to $U_{1}$ or $P_{6}$. In the former case, $U_{1} \subseteq C$ and in the latter $d_{6}(C)>0$. \#

By following the steps of Lemmas 9 and 10, and using similar techniques, we can prove

LemMa 16. If $d_{6}(C)>0$, then $C$ is reducible to $P_{6}$. \#

We now deal with the case where $U_{1} \subseteq C$. Note that if $\sum_{i=2}^{4} d_{i}(C)=0$ and $H \subseteq C$ is isomorphic to $C_{3}$, then there is $H^{*} \cong U_{1}$ such that $H \subseteq H^{*} \subseteq C$. This prompts 
LEMMA 17. If $\sum_{i=2}^{4} d_{i}(C)=0$ and $C$ has just one subgraph isomorphic to $C_{3}$, then $C$ is reducible to $U_{1}$.

Proof. Let $H$, with points $w_{1}, w_{2}, w_{3}$, be the subgraph of $C$ isomorphic to $C_{3}$ and let the cacti $Y_{1}, Y_{2}, Y_{3}$ be rooted at $w_{1}, w_{2}, w_{3}$ respectively (see Fig. 9). Suppose without loss of generality, that $\left|V\left(Y_{1}\right)\right| \leqslant\left|V\left(Y_{2}\right)\right| \leqslant\left|V\left(Y_{3}\right)\right|$. Since $\sum_{i=2}^{4} d_{i}(C)=0$, we have $\left|V\left(Y_{2}\right)\right| \geqslant 2$ and $\left|V\left(Y_{3}\right)\right| \geqslant 3$. We shall prove that $C$ is reducible to $U_{1}$ by induction on $n=|V(C)|$. Note that $n \geqslant 6$. If $n=6$, then $C \cong U_{1}$ and there is nothing to prove. Suppose, then, that $n>6$ and that the lemma holds for all cacti under consideration of order $<n$. Let $k$ be the least $i$ such that $\left|V\left(Y_{i}\right)\right|>i$, such an $i$ existing since $n>6$. By Lemma $2, Y_{k}$ is semi-stable at a non-cutpoint $w \neq w_{k}$. By the choice of $k$, it follows that $C$ is also semi-stable at $w$. Now $C_{w}$ is reducible to $U_{1}$ by the inductive hypothesis, and so $C$ is reducible to $U_{1}$. The lemma follows by induction. \#

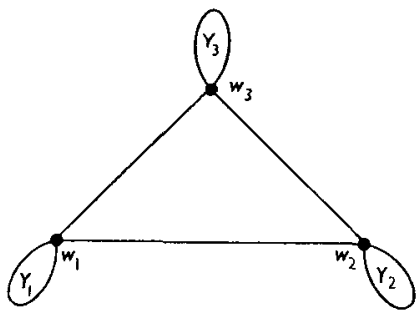

Fig. 9.

The proof of the following lemma is similar to that of Lemma 10.

LemMa 18. If $\sum_{i=2}^{4} d_{i}(C)=0$ and $C_{3} \subseteq C$, then $C$ is reducible to $U_{1}$. \#

From Lemmas 15, 16 and 18, and Theorems 2, 3, 4 and 5 we deduce

THeOrem 6. The cacti in $A_{6}$ are precisely $U_{2}$ and those cacti $C$ such that $\sum_{i=2}^{4} d_{i}(C)=0, d_{5}(C)=0$, and either $C_{3} \subseteq C$ or $d_{6}(C)>0 . \quad \#$

Finally we characterize the cacti in $A_{7}$. In fact, we have already done so, since $C \in A_{7}$ if and only if $C \notin\left(A_{0} \cup A_{4} \cup A_{5} \cup A_{6} \cup A \cup U\right) \backslash\left\{U_{3}, E_{7}, U_{4}, P_{7}\right\}$. Theorems 1, $2,4,5$ and 6 therefore imply

THEOREM 7. The cacti in $A_{7}$ are precisely those cacti $C$ such that

$$
\sum_{i=2}^{4} d_{i}(C)+d_{5}(C)+d_{6}(C)=0
$$

and $C_{3} \ddagger C$ and $C \notin U$ and $C \notin U_{2}$ and $C \nsubseteq U_{5}$. \# 
Since we have determined index-0 graphs to which given cacti in $A_{4}, A_{5}$ and $A_{6}$ optimally reduce, we similarly determine the index- 0 graph to which a given cactus in $A_{7}$ optimally reduces.

First of all, we have

Lemma 19. If $C \in A_{7}$, then either $C \cong P_{7}, C_{8}$ or $U_{4}$, or $U_{3} \subseteq C$ or $E_{7} \subseteq C$.

Proof. If $C \in A_{7}$, then $C$ is reducible to an index-0 graph of order 7. It follows that $C$ is reducible to $P_{7}, U_{3}, U_{4}$ or $E_{7}$. If $C \not P_{7}, C_{8}$ or $U_{4}$, then by Lemma $7, C$ is reducible to $U_{3}$ or to $E_{7}$, so that either $U_{3} \subseteq C$ or $E_{7} \subseteq C$. \#

Finally, by using the techniques of the proof of Lemma 17, we can prove

Lemma 20. If $C \in A_{7}$ and $C_{4} \subseteq C$, then $C$ is reducible to $U_{3}$. \#

The results of the paper can now be summarized in the theorem below which enables us to see the stability index of any given cactus, and a graph to which it may be optimally reduced, by consideration of simple structual properties of the cactus.

THEOREM 8. Let $C$ be a cactus.

(i) $C \in A$ if and only if $C \cong P_{n}, n \geqslant 4$, or $C \cong E_{7}, U_{1}, U_{2}, U_{3}, U_{4}, U_{5}$. These cacti may not be reduced at all.

(ii) $C \in U$ if and only if $C \cong C_{n}, n \geqslant 9$, or $C \cong U_{6}, U_{7}$. These cacti all have stability index 1 and if $C \cong C_{n}$, it is optimally reducible to $P_{n}$, if $C \cong U_{6}$ it is optimally reducible to $P_{8}$, and if $C \cong U_{7}$ it is optimally reducible to $U_{5}$.

(iii) $C \in A_{0}$ if and only if either $C \cong K_{1}$ or $C$ has an essential copy of $P_{2}$ or $P_{3}$. These cacti may be completely reduced.

(iv) $C \in A_{4}$ if and only if $C$ contains an essential copy of $P_{4}$ but no essential copy of $P_{2}$ or $P_{3}$. These cacti are all optimally reducible to $P_{4}$.

(v) $C \in A_{5}$ if and only if $C$ contains a strict essential copy of $P_{5}$ but no essential copy of $P_{2}, P_{3}$ or $P_{4}$. These cacti are all optimally reducible to $P_{5}$.

(vi) $C \in A_{6}$ if and only if $C \cong U_{2}$ or $C$ contains an essential copy of $P_{6}$ or a copy of $C_{3}$, but no essential copy of $P_{2}, P_{3}$ or $P_{4}$, nor a strict essential copy of $P_{5}$. If $C \cong U_{2}$ it is not reducible, if $C$ contains an essential copy of $P_{6}$ it is optimally reducible to $P_{6}$, and if $C$ contains a copy of $C_{3}$ it is optimally reducible to $U_{1}$.

(vii) $C \in A_{7}$ if and only if $C$ is not one of the graphs in

$$
U \cup A_{0} \cup A_{4} \cup A_{5} \cup A_{6} \cup\left[A \backslash\left\{P_{7}, E_{7}, U_{3}, U_{4}\right\}\right] \text {. }
$$

If $C \cong P_{7}, C_{8}$, it is optimally reducible to $P_{7}$, if $C \cong U_{4}$ it is not reducible, if $C \npreceq P_{7}$, $C_{8}, U_{4}$ and does not contain a copy of $C_{4}$ it is optimally reducible to $E_{7}$, otherwise $C$ is optimally reducible to $U_{3}$. \# 


\section{References}

D. D. Grant (1974), "The stability index of graphs", Proc. Second Australian Conference on Combinatorial Mathematics, Lecture Notes in Mathematics No. 403 (Springer-Verlag, Berlin), pp. 29-52.

F. Harary (1969), Graph Theory (Addison-Wesley, Reading, Mass.).

P. Heffernan (1972), Trees (M.Sc. Thesis, University of Canterbury, New Zealand).

D. A. Holton and D. D. Grant (1975), "Regular graphs and stability", J. Austral. Math. Soc. 20, (Ser. A), 377-384.

K. L. McAvaney (1975), "Semi-stable and stable cacti", J. Austral. Math. Soc. 20 (Ser. A), 419-430.

K. L. McAvaney, D. D. Grant and D. A. Holton (1974), "Stable and semi-stable unicyclic graphs", Discrete Math. 9, 277-288.

N. Robertson and J. A. Zimmer (1972), "Automorphisms of subgroups obtained by deleting a pendant vertex", J. Combinatorial Theory, Ser. B. 12, 169-173.

Department of Mathematics

University of Reading

England

Department of Mathematics

University of Melbourne

Parkville 3052

Australia 